\title{
Holistic health: Predicting our data future (from inter-operability among systems to co-operability among people) ${ }^{\text {is }}$
}

\section{Angelo Rossi Mori*, Marta Mazzeo, Gregorio Mercurio, Rita Verbicaro}

eHealth Unit, Institute for Biomedical Technologies, CNR, Rome, Italy

\section{A R T I C L E I N F O}

Article history:

Received 13 February 2012

Received in revised form

25 July 2012

Accepted 17 September 2012

\section{Keywords:}

Holistic health

Data quality

Long-term conditions

Social care

eHealth policies

Care pathways

Patient engagement

Semantic interoperability

Attention Points

Policy-Oriented Health Record

\begin{abstract}
A B S T R A C T
Data depend on processes; processes depend on organizational models; organizational models depend on regulations and policies. This position paper envisages the future data scenarios and the related research needs by addressing this whole logical chain.

A 'smarter health and wellness future' requires the proactive engagement of citizens and of their caregivers, and the cooperation of health professionals across care facilities, with intense usage of mobile communication and connected devices. This ecosystem of people and organizations is currently extremely fragmented. Technology offers the possibility to mediate among the different actors in order to build a functional care team around the specific needs of each individual, i.e. a 'virtual facility'.

However, this requires policies and regulations in every jurisdiction that motivate providers and their organizations to collaborate among themselves and with citizens according to explicit individual plans of care provision, to share their goals and negotiate their respective roles with respect to each citizen.

Once a collaborative organizational context is established within integrated care models, policy makers could identify a critical mass of relevant shared processes and isolate a set of 'Attention Points' with predictable actors, concerns, activities, and thus highly precise information needs. For each Attention Point, a template for a Context-Specific Profile of the patient could be produced, e.g. as an HL7-CDA schema that fully specifies the mandatory and optional (clinical) data useful to support the care processes and to manage governance indicators. In relation to these predictable Attention Points data sources can be aligned to achieve reasonable coherence and consistency. Attention to data quality can be improved in a context of systematic re-use of the same data by different actors in different contexts. From a collection of profiles it could be possible to set up the core of a multi-purpose "Policy-Oriented" Health Record (POHR), shared by the functional care team in the citizen's ecosystem. In fact, the shared management of selected clinical data should be no more based on the a posteriori extraction from the personal notes of each professional, but on the cooperative construction of a systemic resource, together with the administrative and organizational data, able to support the management of innovative, integrated care models.
\end{abstract}

\footnotetext{
This paper originates from the Discussant Presentation in the session "Predicting our data future" at the OECD-NSF Workshop "Building a smarter health and wellness future", Washington, February 2011 [1].

* Corresponding author. Tel.: +39 3346982123.

E-mail address: arossimori@gmail.com (A. Rossi Mori). 1386-5056/\$ - see front matter @ 2012 Elsevier Ireland Ltd. All rights reserved. http://dx.doi.org/10.1016/j.ijmedinf.2012.09.003
} 
In addition, the policy-oriented focus on routine data within a set of predictable situations makes it possible to stratify an appropriate number of citizens into homogeneous classes and to produce timely indicators of processes and outcomes from routine data for governance purposes, e.g. to optimize the allocation of resources, to drive continuous education, or to promote epidemiological studies.

(c) 2012 Elsevier Ireland Ltd. All rights reserved.

\section{Introduction}

Most countries and regions are entering the "connected health" era. Information and communication technologies (ICTs) affect all sectors of our lives, through a range of communication devices and infrastructures (such as mobile phones and Internet) and through selective access to data, information and knowledge. Progress in eHealth research is needed to enable the introduction of dramatic changes in the organization of health and social care; in fact eHealth solutions could assist the move toward more sustainable care systems and effectively further transfer care provision to the community, by supporting home care and reducing unnecessary hospitalizations [1-5].

E-Health policies could become a crucial component of economically sustainable healthcare strategies through the introduction of advanced organizational models enhanced by appropriate ICT solutions. The European Commission is increasingly stimulating eHealth research toward the challenge of promoting a holistic health perspective in order to build a smarter health and wellness future [6], by integrating the various ICT initiatives within the overlapping fields of healthy aging, e-inclusion, ambient assisted living, and social care informatics [7-11].

This position paper deals with the future role of routine clinical data, their usage in the care provision and their quality to support the optimal behavior of the various actors around the patient. We expect that the routine capture and sharing of clinical data will be strongly affected in complex ways: (i) the role of data depends on their intended usage in the actual citizen-specific care process; (ii) within that process, the attitude of the actors toward data capture and sharing depends on the organizational model; and (iii) agreements among organizations and facilities to introduce an organizational model depend on the jurisdiction's regulations and policies. Therefore, to discuss the data future, we must discuss the relations among these different layers.

The change management related to eHealth, which originally concentrated on standards and infrastructures, should in future also encompass collaborative care processes and innovative organizational models, and the regulatory and cultural background. We argue that to try to achieve a complete coverage of semantic interoperability, by precisely formalizing the (clinical) data elements needed for all the potential situations, is an endless and useless task; successful and effective solutions can instead be progressively obtained in a large but enumerable set of contexts, where data nature (with admitted values, constraints and cross-relations) can be carefully defined.

To meet this goal, we suggest that the initial focus should be on the most frequent and predictable 'Attention Points', defined according to health policies so that a critical mass of carefully structured data may be captured, shared, and used by different actors in different processes not only to provide care but also for governance, epidemiology, and research.

\subsection{Organization of the paper}

In Section 2 we first describe the new challenges to eHealth research coming from the current evolution of health and social systems toward innovative and sustainable care models, with a particular attention to disease management, citizen engagement, long-term care. Then in Section 3 we analyze the need to create a shared substrate of structured data, information and knowledge to support the integrated care processes in the citizen's ecosystem, with particular emphasis on the issues of the aging society and long-term care. In Section 4 we further elaborate on the requirements for an effective semantic interoperability, namely to assure the timely availability of all the specific data elements needed to each actor to perform his/her tasks, at least in a limited set of situations.

In Section 5 we claim that a general solution is not viable, and thus policy makers should concentrate on a critical mass of 'Attention Points', i.e. predictable situations concerning shared care or the risk of non-appropriate behaviors, where it could be possible to predict the involved data elements. We also emphasize that an effective data harmonization is a consequence of (i) the organizational integration of the processes for each citizen, and (ii) the level of engagement of the citizen and his/her caregivers. Both factors affect the coherence of the services to promote and maintain holistic health in a daily routine and thus the coherence of the routine data and their re-usability.

Finally in Section 6 we argue that organizational harmonization and process integration - prerequisites to identify the Attention Points and thus to work out which data need to be captured and shared - should be a structural component of a policy to deploy innovative care models in a jurisdiction, based on the cultural background and suitable regulations. It emerges as a central tenet for the comprehensive deployment of a holistic approach to health and cannot be achieved casually by spontaneous evolution or by initiatives limited to the eHealth sector.

\section{Technology-enhanced innovative organizational models}

The health systems worldwide are looking for more sustainable care models, which could improve quality of care and make an optimal usage of the resources. In this section we discuss the potential role of the technologies in the diffusion of innovative care models and, vice versa, the influence of the 
health policies on the correct deployment of the technological innovation and thus on eHealth research.

\subsection{The processes of change management in the health sector}

The evolution of health informatics in the last decades of the past century was mainly driven by a spontaneous process of adoption, independently carried out in several circumscribed environments (e.g. laboratory services, imaging departments, hospital administration and GP practices) [4,12-14].

In the recent years e-government plans in various countries have accelerated the adoption of ICT and eHealth in particular [15]. These plans usually act on two streams:

- national/regional infrastructures to safely share clinical data and documents among care providers (e.g. broadband network, identification/registry of patients and professionals, privacy and security);

- specific eHealth initiatives for certain ancillary services, e.g. e-booking, e-prescriptions, electronic diagnostic reports, patient summaries.

The first stream enables the deployment of the infrastructure for the longitudinal Electronic Health Record (EHR) in large jurisdictions and of the broadband services, e.g. for 'teleHealth' in remote areas.

The second stream mainly concerns 'horizontal' services that replace paper workflows for the population of entire jurisdictions.

In a previous paper [16] we considered both these directions as 'Ptolemaic', because the technological policies lie at the center and the organizational changes 'revolve' around them. We were claiming that it was time to consider a complementary 'Copernican' view, where a third stream is centered on health and social care policies, with technological services revolving around them (even if these services may often act as crucial catalyzers): national and regional care policies imply structural decisions on normative, economic, organizational, logistic, cultural, and educational issues on specific care domains, and should eventually also provide the context and the priorities for a set of appropriate ICT solutions.

Health policies would mostly have a 'vertical' focus on specific targets, e.g. integrated management of a long-term condition, risk control for the frail elderly, support of independent living for those with disabilities, promotion and supervision of health in children's normal growth process. They would be based on an accurate stratification of the target population for each kind of intervention and give increasing attention to promoting a holistic view of each individual. However most decision makers in health care organizations do not yet consider eHealth solutions as an enabling, intrinsic (and ubiquitous) component of current efforts to systematically increase quality and appropriateness in an economically sustainable care system.

As a result, often the current emphasis in the demand for ICT solutions is still on administrative issues and on ancillary services, apart a few exceptions (e.g. [17,57-59]). There is a lack of a shared vision and of adequate investment in means to cope with routine processes of care provision. This bias negatively affects also the vendor side: the market for care support, i.e. for the core business of health and social care, is fragmented and it is difficult to develop and maintain robust products. The eHealth industry is therefore underdeveloped in comparison to the ICT support on other economic sectors. Similarly, while health professionals see the value of some technologies (e.g. diagnostic devices) which are focused on their technical tasks, they often do not see pervasive and integrated ICT solutions as an effective support for their daily work of patient care and therefore do not put pressure on decision makers.

Both administrators and professionals are adversely affected by the lack of awareness of stable and comprehensive informatics best practices (other than initial technology testing trials) and by the eHealth sector's poor ability to generate scientific evidence and to demonstrate consumer acceptance.

The next generation of large-scale eHealth services should not be simply technology-driven: an explicit and systematic research effort should eventually respond to changes in the models of care provision (from hospital to home, chronic disease management, smart elderly health and wellness, integration of social and health care, etc.), with evident benefits for professionals and citizens alike. This will create a new context for widespread data management.

\subsection{Citizens' proactive role in their health}

Interaction of many factors is modifying the context of care provision: demographic changes lead to more elderly people in the population; better care allows more elderly people to live reasonably well and independently at home or in residential facilities, even if frail; care is required for a longer period, usually for permanent long-term conditions $[18,66]$.

Integrated information systems and modern devices may facilitate the engagement of citizens and caregivers, in collaboration with the social and health care professionals, in coping with their long-term conditions, especially when the situation is stable and thus predictable $[12,13]$.

By "caregiver" we mean here any person - without particularly specific skills - that assists a recipient of care, at home or in a residential facility; she/he could be a family member or a volunteer (i.e. an informal caregiver) or a person paid by the family or by the care services (i.e. a formal caregiver). She/he could receive some simple training; she/he could be a direct user of the eHealth services, e.g. for training purposes, for alleviating the burden, or for passing on information to care professionals [19].

In addition to earlier telemedicine, telemetric, and electronic record technologies, a new generation of smart devices can be placed where people live. They are able to perform measurements, set off alarms, or capture videos. These devices can be remotely controlled and can send data to remote places for appropriate interpretation by skilled people $[15,20]$.

A crucial topic for eHealth research may be on the modalities that could enable every actor to perform additional care-related activities, which are currently performed by more skilled people [18]: 
- a number of tasks will be passed from professionals to non-professionals (including the subject of care), under the (remote) supervision of more skilled people if needed;

- a number of subjects of care will be more independent and will require just a limited support by caregivers and technology; their care needs will thus go below the threshold that qualify for the constant intervention of a care professional;

- frail subjects of care will have the opportunity to be more autonomous and safe during their routine activities, including ADL (Activities of Daily Living) and IADL (Instrumental Activities of Daily Living); they will require less support from the non-professional caregivers around them.

This evolution will increase the burden for the citizens and the responsibilities of the subjects of care and their caregivers (patient engagement), and will change the burden on the social services.

However the needs of each individual and the capacity of his/her informal network to provide the required assistance varies from person to person and over time. Hence, we anticipate that an essential research topic will emerge, about the systematic recording (namely within comprehensive information systems and with structured data) of the social conditions that influence the provision of health and social care and the compliance of the subject of care and related caregivers, together with their skills and capacities/limitations, as well the activities performed at home by the subject of care and the caregivers.

Particular attention should be paid to the research on the effects of systemic adoption of domotics and personal devices (for surveillance, alarms, fitness aids). If the use of remote devices will be a key element of care plans and healthy life styles, appropriate provisions will be needed to enable care professionals to effectively manage the related data (perhaps with mediation by call centers).

\subsection{The organizational changes enabled by the appropriate use of ICT}

Smart health and wellness will be at the center of this paradigm change. ICT solutions to enable new models of care organization could have structural effects that go well beyond the isolated effect on citizens' single conditions or compromised functions.

First of all, in a (citizen-centered) 'system' of holistic care, each actor should be aware of the presence, the role and the objectives of the other actors concerned with the subject of care, according to his/her specific plan of care, i.e. there should be an implicit or explicit 'care pact' among all the actors, including the patient. In fact, as the care needs (and thus the facilities and the professionals involved) differ for each recipient, the care system should be functionally configured around him/her. From an organizational perspective, it is therefore important to collect notifications of the relevant activities performed by each professional (i.e. about the contacts of the recipient with the health and care system), by the recipient and by the caregivers. This information can be structured, aggregated or selectively presented to each actor according to his/her specific information needs, subject to the regulations and the citizen's preferences about privacy.

Optimization of care processes may require new job profiles, including the one on the 'care manager', a tutor who interfaces between the citizen and the care system. He/she would have an innovative software toolkit to manage the information needed for performing his/her specific tasks (e.g. communicate with the citizen, be informed about activities performed at home, monitor remote devices, access practical information on available services, book on line and alert appropriate professionals).

In addition to the elements that directly influence care provision, significant effects could be achieved by the generation of indicators (from the routine data captured by the health and social services and by the citizen) to improve the governance of the care system and optimize the allocation of resources for care provision.

Finally, indirect mechanisms that influence information management in the care processes should be considered. These include the education of the citizen and of caregivers and their access to knowledge, regarding, for instance, the management of a long-term condition, the patient's rights, and administrative issues.

Perhaps the most relevant feature of the phenomenon will be neither the increase of ICT solutions and devices on the market nor the ability to better cope with each condition or compromised function (by assisting individuals to cope with care and daily activities). The real impact on the capture, sharing and interpretation of data in the care system could instead come from the deep organizational changes (according to new laws and regulations) that an appropriate data management may enable for the health and social system, in particular to cope with a critical mass of chronic and other long-term conditions.

The above evolutionary scenario can only be qualitative: it is hard to predict, for each country or region and for each kind of technological solution, the future developments about the potential speed of adoption of technology, the care models and the incentives that will be put in place, and the quota of costs and benefits directly attributable to the technologies introduced to respond in the coming years to the pressure by the health sector.

\section{Meaningful use of new and old kinds of data}

New sources of data are gradually emerging in the health and social care sector. Information systems should be able to combine them with data that are in principle already available but not yet fully exploited. The research should provide solutions to yield a coherent, shared information base for each individual.

We will see in subsequent sections that the effectively achievable level of data coherence depends on the degree of organizational integration of all of the actors in the patient's ecosystem (including the patient and the caregivers) and on the predictability of the care situations involved. 


\subsection{The need to manage various kinds of data in the patient's ecosystem}

Collaborative data management in the health and social sector is still largely paper-based or relies on direct human communication, especially when collaboration among professionals with different roles is involved.

Modern technologies offer the opportunity to develop a consistent approach on the "Management of Information, Communication and Knowledge" (which we named MICK in [16]) to create and handle a ubiquitous and pervasive body of structured information, made accessible to authorized persons according to endorsed privacy policies, with pre-set permitted views based on their roles.

However data coherence requires consistent data structures and vocabularies, and services able to re-use the data for multiple purposes. It implies harmonized data capture and processing that involve:

- multiple locations, e.g. clinical and social care facilities, home;

- multiple health and social care sub-specialties;

- multiple types of data, e.g. clinical, organizational, administrative, economic;

- multiple technological contexts, including social networks, email, SMS, web/local questionnaires.

In the next sections we argue that the important achievements of the last 20 years in the standardization of messages, documents and terminology do not yet ensure that the data available to an actor at a given moment can satisfy his/her needs for information to perform specific care activities.

The attention so far is mostly relating to administrative and ancillary workflows or to medical terminology about pathological conditions. They do not necessarily cover the particular data sets [21] required by processes for the effective clinical communication in shared-care plans and for the calculation of indicators for clinical governance and self-audit: detailed specifications of such data sets, agreed at international level and suitable for direct deployment by application developers, are still largely missing.

\subsection{Emerging additional types of data}

The evolution of the patient's ecosystem requires research to comprehensively cope with several additional types of data that are emerging, including:

- data generated by a chronic/frail/dependent citizen or by his/her caregivers as a consequence of his/her engagement, including the data generated by innovative devices outside care facilities: smartphones, domotics equipment, home devices for diagnosis and surveillance;

- data to comply with wellness issues;

- genetic data for personalized medicine.

Furthermore, the potential for new uses of currently captured data should be considered, when compatible with local privacy regulations. For example, the processing of suitably structured data may be used in well-defined contexts to support decisions or to generate recalls or alerts. In particular, indicators for (self-)audit and governance could be derived from the processing of anonymized data from routine documentation, e.g. orders, diagnostic reports (laboratory and images), visit records, records of therapeutic procedures, problem lists, log of contacts with professionals, administrative procedures, booking, or claims reimbursement.

\subsection{Re-use of data requires appropriate representation of details}

Medical and care expressions are very sensitive: an implicit or explicit detail can strongly influence interpretations and actions. Data captured with an appropriate level of explicit details will be suitable for transformation and re-use either for direct care purposes or for aggregation and statistics, e.g. for governance and research [22-25]. Various approaches can be used to manage knowledge and meaning (see also [22]):

- free text is very expressive but not suitable to assure that all the details required for further processing are systematically captured;

- classifications (e.g. ICD) disregard details, to create homogeneous classes for statistics and reimbursement;

- compositional nomenclatures (e.g. SNOMED CT) preserve the details, but are too flexible (i.e. they offer multiple ways to code the same concept) and difficult to normalize;

- instances of Archetypes [26] and Domain Clinical Models [27] formally represent chunks of knowledge that require organization into a context;

- ontologies (e.g. for the semantic web and the Universal Exchange Language proposed by PCAST [28]) in principle will make meanings computable but do not yet allow for pervasive deployment in routine care.

We suggest that the development of a crucial resource on data sets to support semantic interoperability (globally referred to below as the 'Semantic Infostructure', see Section 4.2) should initially focus on predictable procedures in a significant set of scenarios, e.g. to manage data coherence on the booking and selection of the appropriate facility for organizational purposes, or to handle explicit decisional criteria in the reference clinical pathways for clinical purposes.

The priorities in developing the methods for the harmonization of terminologies, coding schemes and archetypes across heterogeneous environments should concern the predictable processes in the most relevant situations and should be determined through the analysis of the data elements required by these procedures; the level and granularity of details to be captured should be the one required by the most demanding procedure among them.

\subsection{Amalgamating data, information and knowledge into a comprehensive substrate}

At present care processes are often fragmented, and there is an increasing need of research to interrelate them. To integrate social and health care effectively, all interconnected information resources should in principle be coherent across each area of responsibility (see Fig. 1). At some point in future all 


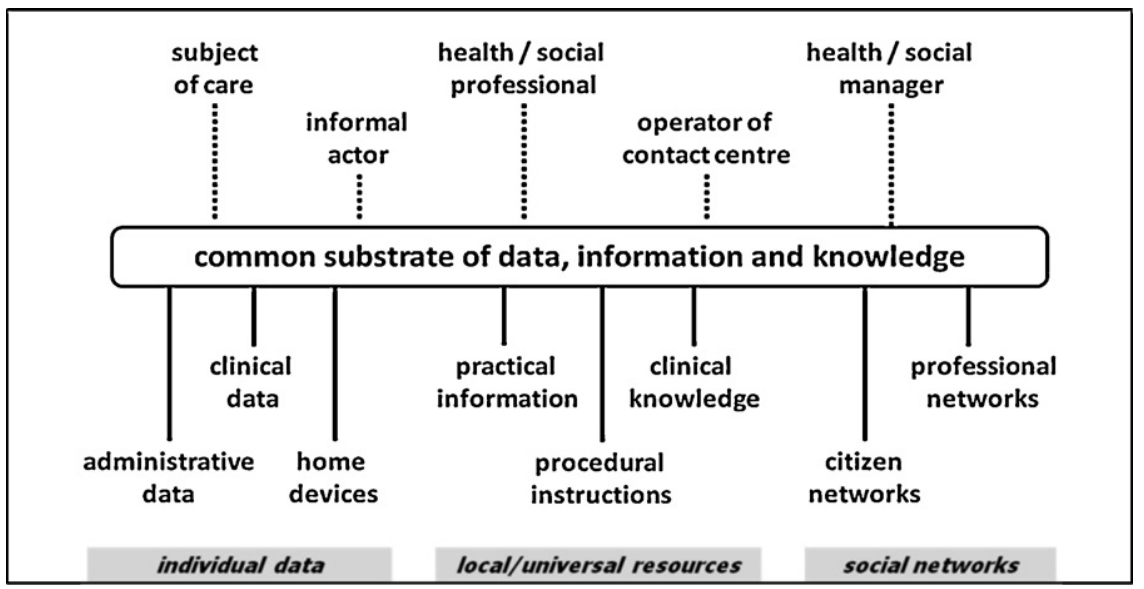

Fig. 1 - The eHealth environment with a shared information substrate [16].

actors will necessarily ask to rely on a shared substrate for the Management of Information, Communication and Knowledge (MICK [16]).

This substrate will hold all the relevant data, information and knowledge available within the care system and should be able to select and forward to all involved actors the data that are relevant to their role, compatibly with specific policies concerning notification and organization of information. The transformations for multiple usages of information require an intense use of a Semantic Infostructure.

\section{Consistency of data from heterogeneous sources}

A partial data coherence and consistency among heterogeneous sources can be achieved under current standards for information models (e.g. HL7-RIM [29]), data structures (messages and documents, e.g. ANSI-HL7-CDA [38]) and data elements (e.g. HL7 Detailed Clinical Models [27], EN13606 OpenEHR Archetypes [26]).

In this section we assert that most standardization activities actually fit processes related to ancillary services, but are less effective for the core business of the care milieu, i.e. collaboration on care provision.

\subsection{Subordinate us. parallel responsibilities}

We shortly recall here a previous discussion on this topic [16]; as depicted in Table 1, we called the case about ancillary services as 'activities with subordinate responsibilities' and the case about collaboration on care provision as 'activities with parallel responsibilities'.

In the first case, a physician with responsibility for the health-care action (i.e. with a 'care mandate' according to [30]) may involve another professional by an 'order' to perform an auxiliary activity, e.g. a diagnostic service. This order implies the transfer of a partial responsibility, which limits the autonomy of the subordinate decisions. The request triggers a workflow and normally should result in a report.
This kind of communication is historically structured enough to be supported by current interoperability measures. Note that 'interoperability' may be considered as a contraction for "ability to interoperate" or, in this context, "ability of health information systems to interoperate".

In the second case, several healthcare professionals (and the citizen with his/her caregivers) have complementary roles for diverse aspects of the care provision. An explicit shared plan or a more or less strong 'care pact' may formalize their cooperation. These activities involve the "ability of people to cooperate", which we called 'co-operability'.

Co-operability adds further constraints to interoperability. It involves the need to assure the proper capture and the timely availability of the specific clinical data elements needed by each actor to perform his/her tasks at a particular moment or to interact with other actors [31-34]. These data elements are highly dependent on the context (phase of the clinical pathway, other conditions of the patient, kind of facility concerned, etc.).

\subsection{Co-operability standards - data sets and the 'Semantic Infostructure'}

Standard specifications to support co-operability should build on technical standards and should require a strong involvement of the professional care milieu. Earmarked research should envisage how they should formalize, in a format suitable for effective management by ICT applications, different kinds of knowledge, which together constitute the 'Semantic Infostructure' that makes it possible to benefit from semantic interoperability [3,35-37].

They should formally describe not only the (clinical) data elements needed in each particular situation, but also the related processes, i.e. the actors involved with their typical concerns, tasks and mutual roles. It is therefore impossible to formally describe all of a citizen's potential health-related situations. However, a significant number of predictable situations can be represented within reference care pathways, with the mutual responsibilities of professionals agreed and their interaction points made explicit [34], and with context-specific needs for information and communication. 
Table 1 - Comparison between subordinate and parallel responsibilities (adapted from [16]).

Activities with subordinate responsibilities

Connecting systems; interoperability among systems

Activated by an order, prescription

Prescription, medical report, discharge letter, organizational activities, e.g. e-booking

Modalities of interaction historically tested and fixed, with a consolidated usage of paper forms

Partial mandates, referring back to the issuing

physician; bureaucratic procedures

Predefined paper or electronic forms; messaging

standards
Activities with parallel responsibilities

Connecting people; "co-operability" among people

May be independently activated

Multi-disciplinary evaluation team, often across different organizations (e.g. GP, hospital, ambulatory care)

Flexible modalities of communication, depending on the clinical context, often just partially formalized Distributed responsibilities among professionals and proactive role of the patient and care helpers Multiple "local" modules or very specialized ones (scores and scales); advanced Semantic Infostructure
Many existing descriptions of clinical pathways involve the chronic disease management for single defined conditions. Other pathways specify the ideal behavior to assure the quality of care in situations at risk for non-appropriateness. This approach could also provide the background for managing the data in a personalized plan of care for an individual with comorbidities and a specific domestic setting.

However, no description is known to the authors that provides implementable specifications, according to international standards, on services and data for software developers.

Therefore an effort could be foreseen, to formalize a set of 'Attention Points' in the context of most of these pathways, to respond to precise information needs, by defining the standard specifications for a suite of eHealth services based on structured data [35].

In fact, only if the right data elements are properly prearranged in an agreed computable format way will it be possible to cope with the urgent need for the integration of social and health-care processes and the patient engagement, enhancing quality and meaningful use of widespread, semantically interoperable EHR systems.

In addition, well-organized routine data will bring new opportunities for governance and reimbursement mechanisms for integrated care, with the timely production of comparable indicators of process and outcome, uniform across large jurisdictions and - eventually - at international level.

For each Attention Point, the professional community should describe actors, roles, concerns and tasks, working out a list of the data elements to be captured, stored or communicated for each particular task and thus producing the related standard specifications. In particular, it should locate two kinds of potential Attention Points:

- the interaction points among the actors [34] in order to identify the task-specific clinical documents for each exchange, with their predictable structured content. That is, it should produce a catalog of potential documents and an explicit list of the expected data elements for each document, i.e. the earmarked (clinical) data set [21] to be communicated when transferring responsibilities in the course of a plan of care.

- the appropriateness points, where one or more actors should capture the data set needed to provide the optimal services according to the health policies.
In addition, the professional community should produce a series of earmarked terminological subsets, i.e. the value sets for each field in a task-specific data set.

Finally, to improve data quality it would be helpful to formalize the knowledge needed:

- to validate the data during the input procedures through a list of "normal values", as well as through a list of the values that are "expected-in-context", i.e. the values that are accepted in conjunction with other parameters;

- to double-check the data in the various contexts of their re-use;

- to assure quality in specific decisions, interactions and indicators.

All together, the above specifications will make up the core of the standard Semantic Infostructure to cope with the selected Attention Points.

\subsection{A document-based approach, with constraints at level 3 of usage of HL7-CDA}

To ensure an effective co-operability, all of the above material should be harmonized across specialties and made available to software developers, to be gradually embedded into the operational systems. International specifications should accommodate local variations.

This approach is compatible with the most frequently used standard for the representation of clinical documents (i.e. ANSI-HL7-CDA Error! Reference source not found.), which can be used according to three levels of predefined detail:

- Level 1 provides the metadata of the "header" that describes the document as a whole (e.g. event, patient, clinician) and the generic XML labels to distinguish the nature of the internal artifacts of the "body" (sections, text sentences, codes, etc.), without any specific clinical meaning. It allows for simple queries to retrieve and list the documents in a collection. This level is represented by the usual ANSI-HL7-CDA Clinical Document Architecture ("level one").

- Level 2 concerns the explicit CDA constraints for naming and structuring the subdivisions of a clinical document (e.g. patient history, family history, active problems, diagnostic results and procedures performed). Standards in this field are converging, e.g. the ASTM-HL7 implementation guide 
on the CCD Continuity of Care Document [39] specifies the title and the code for each section and the data structure of the clinical data items that are expected to appear within each section.

- Level 3 provides the actual data elements and specifically agreed indicators (with the related terminologies), i.e. it specifies which (clinical) data should be present in a particular kind of document in a given circumstance, depending on the context. As discussed above, this is the level of semantic interoperability needed to cope with the in-depth co-operability among professionals, in particular across organizations and beyond language barriers, even if it will be hard to obtain a large amount of formalized knowledge in a short time period in several Attention Points.

To make further progress on effective support for holistic care, the Semantic Infostructure should contain a significant amount of level-3 specifications, according to the requirements emerging from clinical pathways and patient engagement.

For each Attention Point, a template for a document that describes a Context-Specific Profile of the patient (CSP) could be produced. The template could be an HL7-CDA schema with level-3 specifications, i.e. a set of constraints on the CCD schema that fully specify the mandatory and optional (clinical) data relevant in the context of the referred Attention Point.

The collection of the CSPs of the same patient, about the evolution of the patient's conditions observed at different Attention Points, will build a shared "Policy-Oriented" Health Record (POHR).

Note that the schema for a Context-Specific Profile is more detailed than the schema of CCD or than the one of a generic Patient Summary [67], because it specifies the precise data that should be contained, possibly also with the constraints on the admitted values in the particular context of the Attention Point where it is intended to be applied.

A global catalog of the processes, the services, the datasets, the CSPs (and of their inter-relations) could be a useful tool for an international effort of systematization.

A comprehensive, harmonized ontology made from the data elements that belong to the various data sets made explicit in the CSPs could be used as the reference for all the local adaptations and for the widespread development and adoption of the related Archetypes [26] and Detailed Clinical Models [27].

\section{Predictable data in predictable Attention Points}

We argued in the previous sections that the research and the standards to improve the semantic coherence of shared data should cope with the management of the Attention Points within predictable collaborative processes, e.g. within stable individualized care plans derived from a set of reference clinical pathways, where the attention to the re-use of structured data could stimulate to validate them and to prevent the hazards arising from inaccurate or missing information.
Stable care processes for healthy aging and wellness represent the most promising starting point for a strategic approach to 'connected health' initiatives. This is the situation of many (older) people with some chronic limitations and 'normal life' within these limitations. The role of health care and social care is to manage risks, minimize adverse effects, and compensate for these limitations [40-42].

The World Health Organization defines 'health' as "A state of physical, mental, and social wellbeing, and not merely the absence of disease." [43] This definition therefore includes the maximum achievable level of independent living, which may be facilitated by family, friends, or formal services.

Chronic conditions and permanent impairments put a large share of the burden on the care system (on the primary care and social care professionals, on the citizens and their caregivers, and on avoidable hospital stays). Optimization of support through optimized data, communication and processes may contribute significantly to the quality of care and the sustainability of the sector [44-46].

Long-term care implies organizational issues very different from those for acute hospital care or short-term rehabilitative care. The engaged citizens and their caregivers, perhaps supported by a new generation of home devices and telemedicine services, will manage most routine care at home or in longterm facilities; in addition, patients and caregivers need to know how to recognize relevant events, either in the citizen's state or in their capacity to continue their assistance, that require the alerting of a professional, and to know what to do and whom to advise.

\subsection{Predictable clinical pathways and social interventions as use cases}

A large amount of specific standard subsets of data elements need to be timely available for use in an adequate number of Attention Points about care provision and self-care, e.g. for clinical decisions, care-related interactions, administrative procedures, facility management, and research.

For each applicable citizen as subject of care, each actor should have an explicit role in the shared care plan, with specific goals and targets, with a specific vision of the health issues related to his/her role and the tasks to be performed, with production of the related documentation $[30,31,33]$.

By analyzing several use cases on the different phases of a significant set of 'steady states' for chronic conditions and self-care situations, it is possible to systematically describe the above features and thus to characterize the eHealth services that could support the related processes in different regulatory and practical situations. This effort can create the first set of 'Motivated Operational Frames' (MOFs in [11]), i.e. the part of the Semantic Infostructure related to a particular Attention Point and addressing a specific Health Concern, with the description of a critical mass of coherent modular eHealth services based on structured data, to start a process of progressive systematization of the sector.

The collection of use cases formalized as MOFs can provide the criteria to focus on data elements to be managed within a predefined context and to effectively share them: 
- who should produce each data element, at which moment, in which context,

- to whom, at what moment, for what purpose to make it available.

The analysis of a MOF can identify the parameters that may indicate the need to move to a different steady state foreseen in the same plan of care and thus may trigger a notification to a suitable actor or may require a major revision of the ongoing plan of care.

We stress that an accurate prediction of specific (clinical) data requirements (i.e. the production of the schema for a Context-Specific Profile) is only possible within predefined MOFs. By analyzing the phases of a significant set of Attention Points, it is possible to set up a corpus covering the above features in a systematic way for high priority scenarios and thus to characterize the eHealth services and the data that could support the related processes in a given regulatory and practical context.

There are already several clinically oriented initiatives in this respect (e.g. Map of Medicine Healthguides/NHS Choices [47], or 'Procesos Asistenciales Integrados' [48]). However they rarely reach the level of standard specifications on the structured knowledge (e.g. the HL7-CDA schema for each Context-Specific Profile) that can be directly used by software developers at the Attention Points, to build effective user interfaces for data capture and visualization, as well as to produce standard messages and standard clinical documents.

\subsection{Enabling a virtual care team to behave as a system}

According to emerging organizational models in care provision and health promotion - especially in relation to healthy aging, wellness and long-term care, where "Social Care Informatics meets Health Care Informatics" [49,50] - the multiple actors concerned with a given care recipient should be mutually aware of their reciprocal roles, goals and planned tasks, and should be notified - as necessary - about contacts with the other actors, the activities performed and the data collected [30].

Most eHealth policies are said to be directed toward continuity of care with a "citizen-centric" approach [51]. However, they often seem to rely on the technology-driven infrastructure for unified collection of documents from multiple sources (the longitudinal EHR approach) rather than aim to make the healthcare system a coherent entity in front of the citizen (with an approach that involves the orchestration of activities).

Research is needed to move in that direction and develop the new perspective. To this respect, the CEN standard on continuity of care [30] emphasizes three main concepts:

- the notification of care mandates - WHO is involved?

- the notification of contacts - WHICH ACTIVITY is being performed?

- the notification of health issues (or problem/concern) WHY is this being done?

The latter, if related to an Attention Point, could in future be better described as:
- the notification of the concern activating a Motivated Operational Frame [11] that entails the vision on a coherent set of activities, responsibilities and services for a specific objective - IN WHICH CONTEXT is this being done?

Unfortunately, eHealth policies of most jurisdictions do not explicitly mention those concepts. The systematic management of these data, properly selected and organized for each role, could help to organize efficiently the documentation avoiding an information overload; e.g. the profile of each kind of actor may define which alerts, reminders or reports can be sent by default in push mode to mobile phones or by email.

Suitable eHealth services may use these notifications to control the proper settings to better protect privacy and to enable all the actors to "behave as a system" in the citizen's ecosystem, with a true holistic citizen-centric vision [52]. In fact, each care recipient has specific care needs, which require the participation of a particular subset of professionals, among the ones available across the whole system; they may belong to diverse organizations and be in different locations. Therefore a different, earmarked "functional care team" should take shape around each care recipient and his/her needs, as a kind of "virtual facility" (for an early discussion of the virtual care team, records, and communication, see [52]). Moreover, the citizen should have, as far as possible, a proactive role in the virtual facility, as a responsible participant in the management of his/her health (patient engagement) $[16,37,53-56]$.

\subsection{The multiple usage of structured data}

The adoption of use cases derived from a set of relevant Attention Points could allow for the predicting of the contexts in which the data should be captured and may be transformed to be re-used or shared.

The comparison of the data elements across the data sets formalized in the context of different MOFs will permit to work out unnecessary differences and hidden ambiguities. A systematization activity should allow to produce a combined list of data elements.

Moreover, in principle each Attention Point should deserve a specific content for the related documents; however after the analysis in Section 5.1 similar Attention Points could be detected, to reach a compromise position on a unified structure for a problem-oriented, focused profile, as a generalization of a set of similar Context-Specific Profiles, e.g. for a diabetic patient, an oncological patient, or a frail elderly person, or a referral letter for a specialist consultation.

In addition, a set of services could be identified within a MOF to re-use structured data, within the privacy constraints, in administrative, organizational and governance processes to give appropriate returns to each actor (including the citizen and the caregivers) for the capture and multiple usage of high quality data.

The multiple usages of data could be mutually reinforcing up to a significant number of functions [16]: 
- to share core data between GPs and specialists, as well as between citizens and professionals, on the basis of an integrated care approach (e.g. chronic disease management);

- to enable the GP's self-audit and benchmarking with colleagues, with appropriate predefined adaptable filters, drill-down functions, and reminders;

- to extract timely indicators of processes and outcomes at local and regional level, both for administrative functions and for governance purposes (e.g. reallocation of resources, definition of training needs and education material, change management);

- to provide a kind of continuous medical education to the professionals, based on the routine use - through the software - of tables derived from the authoritative clinical pathways, thus delivering chunks of knowledge-in-context;

- to feed clinical databases for basic epidemiological studies.

Note that the aggregation of data (for self-audit, governance, epidemiology) may be particularly effective for predictable processes in stable and frequent situations, where the conditions of several subjects of care are reasonably similar, so that the clinical, organizational, economical detailed data can be properly structured, inter-related and automatically processed as coded data to yield a statistical significance.

\section{The quality of data depends on policies and organizational models}

\subsection{Healthy aging and wellness}

A comprehensive eHealth environment should be able to facilitate citizen-centered integrated care, i.e. it should cover the whole holistic ecosystem of the citizen's health and social care.

In particular, eHealth implementation plans, synchronized - according to the respective regulations - among national, regional and local authorities, payers and care-provider organizations, should help address the needs of elderly persons who face issues arising from frailty, long-term conditions, mobility problems, or other concerns that limit their Activities of Daily Living.

The elderly may require additional support, often over and above normal healthcare, to ensure their nutrition, personal and domestic hygiene, daily living tasks, and social inclusion.

While the prime source of such support is usually the family, along with neighbors and the local community, formal responsibility for ensuring citizens' safety and well-being and for complementing their informal supporters often lies with agencies under local and regional governance.

An interesting set of research challenges arises from the fact that for some activities the formal service is the sole provider (such as giving an injection), while for others the formal service is a back-up provider when the patient or the family cannot ensure the activity. Two issues arise from this situation involving different contexts for information management: (i) some patients can become "experts" and undertake procedures otherwise performed by formal carers (e.g. self-administered insulin injection) and (ii) the need to think about the health and needs of caregivers linked to the patient's plan [19].

As the need to support an aging society increases, so does the need for closer integration of care. This may be achieved, in part, through the research on integration, or interaction, between ICT policies for health and social care and the stimulation of collaboration and innovation on a broad international scale.

It is worth noting that new high-speed mobile applications, connected devices, social networks, and related cutting-edge smart technological developments can provide unique and unprecedented opportunities for developing an electronic 'Personal Health Assistant', i.e. an 'avatar' of the citizen, made of eHealth solutions in a virtual environment that can help him/her to address the health and wellness challenges of an aging society.

\subsection{The citizen's involvement and quality of data in a Personal Health Record}

The quality and usefulness of data heavily depend on the (perceived) effectiveness of the eHealth services that generate them, and on the organizational background that promotes awareness of the reciprocal information needs of the various actors.

The success of a citizen-centered information system may be expected to reflect the organizational cohesion within the citizen's ecosystem and the citizen's involvement in health maintenance.

The Personal Health Record (PHR) of highly cohesive providers, such as pervasive Healthcare Maintenance Organizations (HMOs) (e.g. Kaiser [57], Veterans Administration [58], Maccabi [59]), is built upon high-quality, care-related integrated information systems.

However, several PHR systems do not correspond to an integrated care ecosystem. Most citizens seem unable or not willing to 'unify' their disparate care experiences in a PHR, as demonstrated for example by the low level of adoption of Google Health:

"When we launched Google Health, our goal was to create a service that would give people access to their personal health and wellness information. We wanted to translate our successful consumer-centered approach from other domains to healthcare and have a real impact on the day-to-day health experiences of millions of our users.

Now, with a few years of experience, we've observed that Google Health is not having the broad impact that we hoped it would. There has been adoption among certain groups of users like techsavvy patients and their caregivers, and more recently fitness and wellness enthusiasts. But we haven't found a way to translate that limited usage into widespread adoption in the daily health routines of millions of people." [60]

In other words, the lesson of Google Health demonstrates that the mere availability of a tool to collect and store data is not a sufficient condition to assure a pervasive adoption. A successful Personal Health Assistant may need to be a consequence of a 'care pact' involving the citizen and the formal 
and informal caregivers: the citizen actively 'takes care' of his/her health and is embedded in an innovative model of care, in which the coherent organization of social care and health care is synergic and drives the introduction of ICT solutions. Engaged citizen and caregivers are the primary source of data and legitimate users of the PHR. The data that they produce properly filtered, assessed, and assimilated by the care professionals - should be considered an integral component of the PHR.

\subsection{A culture of 'systemic' data sharing}

Within the communities were care providers are not accustomed to collaborate (and thus to share data), we claim that an innovative care model requires a deep cultural change. The culture of data sharing should in fact be 'systemic' (i.e. promoted by the organizational model), as it is not possible to rely on spontaneous uptake. Hence this approach can optimally take place in specific settings for any circumscribed Copernican situation that involves the deployment of precise policy-driven organizational innovations (e.g. integrated care, chronic disease management).

A positive attitude to data sharing could be the result of strong organizational cohesion (as in the HMOs mentioned above), where collaboration and data sharing are an integral part of the culture of all of the enterprise's employees and collaborators. Data are managed as part of the organization's inclusive information system.

Some data sharing could also be enforced through the management of multiple interacting facilities (e.g. the US Health Information Exchanges [61,62] and similar bodies in other countries). Patients could be stratified or classified according to the care tasks involved and insurance payments (e.g. Medicare, Medicaid) could be based on the underlying expected comprehensive care processes [63]

A motivating program (not necessarily direct payments) could involve the virtual 'functional team' composed of all of the actors contributing to the care of a given patient (professionals, caregivers, patient), who later negotiate how to share the benefits of the program. In this case, targeted collaboration across the facilities will be the main objective of the program; the sharing of structured data could be a secondary, facilitatory objective. At present, most successful data sharing initiatives are related to the effective use of ancillary services with subordinate responsibilities (see Section 4.1), e.g. e-booking, e-prescription, diagnostic tests (orders and reports). In such cases, there is already a kind of 'contract' between the parties. However, this mechanism cannot be simply extended to sharing all the data generated in a context of parallel responsibilities (see Section 4.1), during an actual care activity by professionals, caregivers and citizens (perhaps using home devices).

The motivation to communicate and share data is an important, yet somewhat overlooked point. Discussion has tended to focus on privacy and connectivity among systems, as if co-operability could be a given once technical and legal barriers are removed; yet every communication implies a social contract that must be negotiated within the related cultural environment.

\subsection{The influence of the policies on the quality of data sharing}

According to our vision, the policies of each jurisdiction, with their regulations and payment mechanisms (the economic layer), determine the agreements among the various organizations in charge of shared care provision (the organizational layer) and thus the effectiveness of the information services in the care provision (the clinical layer).

The quality of the clinical and organizational data is a consequence of the cohesion among the providers and of the coherence of the information services, as supported by the information system.

The economic layer concerns the agreement(s) between payers and provider organizations. Several mechanisms can be put in place: at one extreme, payment may be separate for each individual act (with the production of detailed administrative data); at the other extreme the payer could consider that the 'unit of payment' is the integrated care as a whole for a class of citizens and for a given period. This implies defining the minimal amount of care services to be provided and setting up a system of indicators of process and outcome derived from the routine data - to verify the quality of the care provided; these agreements could be enforced by law or regulations or could be embedded in the "internal DNA" of an HMO.

The organizational layer concerns the agreements among different provider organizations. In a strongly cohesive environment (e.g. in comprehensive HMOs) individual providers are culturally prone to cooperate and share data. In the case of multiple organizations providing care to the same patient, if the unit of payment is the integrated service as a whole, the managers of the provider organizations (and the individual care providers that do not belong to any organization) must explicitly agree about how the professionals should cooperate in care provision (and how to share reimbursements). We argue that, if cooperation among professionals (and the consequent data sharing) depends on a spontaneous choice by each individual professional, it will probably not occur, even if a "perfect" workflow management system is in place (see Section 6.2).

In a model of integrated care - regardless of the modalities of deployment - the clinical layer is strictly related to the organizational and administrative layers, and requires a positive attitude toward cooperation among the actors in the (virtual) care team, including the citizen and the caregivers. All the actors behave as a virtual, functional team which is built according to the mix of the citizen's conditions, their stages, the complications, the social needs, the local context and the citizen's specific choices of professionals that he/she wants to involve. In principle the professional team is different for each multi-pathological patient. Data sharing should be an intrinsic component of this model.

Ideally a 'contract' could 'enroll' the patient with a stable long-term condition in an explicit plan of care for a reasonable period ( 6 months or one year) and clarify the services that will be provided to the patient by each professional in the functional team and their goal, as well as the role and the targets of the citizen and of the caregivers. The citizen (and the caregivers) could be encouraged to collaborate on the care and 
fulfill specific tasks relating to documentation (in the PHR). We assume that a contract is essential for successful data sharing in that context.

\section{Conclusions}

In principle the analysis of any plan of care should allow for defining the tasks of each actor, the interaction points and thus the potential supporting eHealth services, but it is hopeless to repeat the analysis for any particular individual plan. However, for a number of relevant, predictable Attention Points (as identified by the policy-makers) it is possible to work out a series of Motivated Operational Frames (MOFs) and thus the detailed requirements regarding the structured information, which can then be adapted to individual cases. This effort will support an effective semantic interoperability, by producing the standards on the Semantic Infostructure that can be applied by software developers: on the appropriate clinical data to be captured and exchanged, on the level of coding needed for further processing, on the timely indicators for governance and other secondary uses.

In fact, many fragmented eHealth solutions exist to support the needs of care provision for smart health and wellness, along two directions: on one side, the technology-driven horizontal approach of e-government and current eHealth Roadmaps; on the other side, the problem-driven vertical approach to deploy health and social care policies for specific target groups, with embedded eHealth solutions.

A comprehensive framework to systematically put the two different approaches together is still lacking. To fill this gap, we suggest an (international) initiative to focus on the Attention Points related to the major chronic diseases and the major impairments to daily living activities, which are relevant health problems for citizens and the most resource-consuming segment of the health and social care environment. The main focus should be on the core of Semantic Infostructure that enables or supports the introduction of innovative models of care (e.g. disease management, chronic care model, patient engagement) to deal with the increasing need to integrate health and social care $[49,50]$. That initiative should consider the following three layers of integration.

\subsection{Integrated "vertical" policy-oriented roadmaps}

A significant set of (evidence-based) MOFs could be formalized, specifying the actors involved, with their objectives, roles, tasks, interaction points, as the context to produce a detailed specification on the related information needs. Each of them would constitute the context to situate, compare and rank the wide spectrum of clinical, organizational, managerial, educational, administrative, practical issues, and thus to characterize the ad hoc suite of eHealth services required, with a particular attention to the data elements to be captured and shared, as well as to the governance indicators. The initial focus would be on steady phases of the main long-term conditions and a number of situations at risk for non-appropriateness.

The computable descriptions of eHealth services (including also data elements and accepted values, represented in the standard schema of a Context-Specific Profile of the patient) can afterwards be harmonized into a comprehensive Semantic Infostructure.

The preparation should take into account the implicit or explicit regulations, agreements and established customs in each jurisdiction, to build a comparative framework about health and social policies, funding models, demographic changes and need for care, where the variants of the vertical components of eHealth roadmaps may be positioned.

\subsection{Integrated control and governance}

Integrated care in a fragmented system requires economic agreements (e.g. incentives and payment mechanisms) between payers and providers. For example, a payer could consider a defined period of integrated health and social care for a specific condition as the unit to be 'paid' (a DRG-type of approach).

A suitable regulatory/economic context could be the key to capturing and sharing coherent data, a goal that is not achievable by the eHealth initiatives that focus only on data and infrastructures and rely on spontaneous, non-systemic interactions where individual providers have no incentive to collaborate or to engage citizens.

Control of the actual care provision to individuals and the governance of integrated care in a jurisdiction require indicators of quality and appropriateness, which can also be used for benchmarking and self-audit of care professionals and care provider organizations. This context of systematic re-use of routine information could reinforce the propensity to the quality and the completeness of the data.

\subsection{Integrated management of information in the citizen's ecosystem}

Wellness and long-term care involve organizational issues very different from those for acute (hospital) care. Citizens should be properly engaged in the management of their health and wellness and their caregivers, with the support by a new generation of home devices and telemedicine services, in order to effectively manage most routine care at home or in long-term facilities.

A proactive citizen and the caregivers and professionals caring for him/her - with their mutual relations - comprise a particular 'functional care team', i.e. an ad hoc 'virtual facility' that is different for each citizen, because it depends on the (multiple) health issues, the social situation and the local context that constitute the specific ecosystem of each citizen.

As a consequence, data processing is not determined within the boundaries of each care facility (e.g. by the care tasks normally performed in a hospital ward), as in acute care, but should be arranged 'on demand' according to the comprehensive needs of the functional care team. We argue that, by focusing on the Attention Points enforced by the policy makers according to the local priorities, it would be possible to adopt an ad hoc suite of eHealth services to manage the meaningful use of structured data for a large portion of the routine activities of each functional care team. Data will be presented within structured documents according to standard schemata for each Attention Point (i.e. the Context-Specific 
Profiles of the patient). The processing of the data contained in the Context-Specific Profiles allow to obtain a Policy-Oriented Health Record for each patient to support innovative, integrated care models and the governance of the care system, at least for the set of care processes considered as the most relevant by the policy makers.

\section{Author's contribution}

Contribution of authors, namely ARM, MM, GM and RV, have been made in Chapters 1,6, and 7; Chapter 5 and literature/web search; Chapter 2; and Chapters 3 and 4, respectively.

\section{Competing interest}

ARM is scientific supervisor for DS Medica and RV is consultant for DS Medica.

\section{Acknowledgments}

The work was partially supported by the European Project ANCIEN - Assessing Needs of Care In European Nations (FP7 HEALTH-2007-3.2-2, Project \#223483) [64].

The activities were partially performed in the context of a Collaboration Agreement with the Italian Ministry of Health, General Directorate for Information Systems; however the concepts expressed in this paper reflect the vision of the authors and should not be intended as a position of the ministry.

The Authors had also the opportunity to discuss this topic and develop stimulating visions within the eHealth ERA [15] and RIDE [4] FP7 European Projects, within the Task Force on Sustainable Telemedicine \& Chronic Disease Management of the European Health Telematics Association (EHTEL) [41] and within the IGEA Project (by the Italian Ministry of Health) on Disease Management for Diabetes [65].

The paper benefited from the Exploratory Workshop on Social Care Informatics and Holistic Health Care: "Social Care Informatics Meets Health Care Informatics - a Holistic Citizen-Centric Vision for Information and Communication Technologies to Support Personal Health", for the European Science Foundation, Keele University, UK, July 2010 [49,50], and from comments and suggestions provided by Prof. Michael Rigby on earlier versions of the manuscript.

\section{REFERENCES}

[1] OECD-NSF Workshop "Building a Smarter Health and Wellness Future", Washington. http://www.oecd.org/sti/smarterhealth (accessed 15-16.02.11).

[2] I. Iakovidis, Introducing Information and Communication Technologies into Medicine: New Challenges for Research and Development, The European File. eHealth in Europe, 2009, pp. 21-23.

[3] A. Rossi Mori, et al., Towards a European roadmap for achieving eHealth interoperability, in: eHealth Berlin Conference 2007-04-18, Special Interest Session II "Electronic
Health Records and Interoperability", Co-organised by the RIDE Project and the EuroRec Institute, 2007 http://www.eurorec.org/news_events/newsArchive.cfm? newsID $=142$

[4] A. Rossi Mori, et al., RIDE D4. 3. 1 - "Policies and strategies", in: Deliverable of the EU Coordination Action "RIDE - A Roadmap for Interoperability of eHealth Systems in Support of COM 356 with Special Emphasis on Semantic Interoperability, 2007

http://www.srdc.metu.edu.tr/webpage/projects/ride/ modules.php?name=Deliverables, http://www.srdc.metu.edu.tr/webpage/projects/ride/ deliverables/RIDE-D4.3.1\%20policies\%20final\%20v06a.doc

[5] Scottish Executive, Building a Health Service Fit For the Future Volume 2: A Guide for the NHS, 2005 http://www.scotland.gov.uk/Publications/2005/05/23141500/ 15035

[6] European Commission (2011). "More years, Better Lives The Potential and Challenges of Demographic Change". Recommendation (2011/413/EU), http://ec.europa.eu/ information_society/activities/einclusion/deployment/jpi/ index_en.htm (accessed 11.07.11).

[7] Council of the European Union (2007). "Joint Report on Social Protection and Social Inclusion", 2007. http://ec.europa.eu/employment_social/social_inclusion

[8] European Commission (2008), Telemedicine for the benefit of patients healthcare systems and society, COM (2008) 689.

[9] European Commission (2009), Telemedicine for the benefit of patients, healthcare systems and society, in: Staff Working Paper SEC(2009)943, June 2009.

[10] European Commission, ICT Research. The Policy Perspective. Report from Information, Society \& Media Unit, 2010.

[11] A. Rossi Mori, G. Mercurio, Verbicaro R, Enhanced policies on Connected Health are essential to achieve accountable social and health systems, Eur. J. ePractice, No. 15 (2012), Special Issue on "Policy lessons from a decade of eGovernment, eHealth \& eInclusion". http://www.epractice.eu/en/journal/issues/

[12] European Commission (2007). "Accelerating the Development of the eHealth Market in Europe". eHealth Taskforce report 2007.

http://ec.europa.eu/enterprise/policies/innovation/policy/ lead-marketinitiative/eHealth/index_en.htm

[13] A. Rossi Mori, G. Freriks, A European perspective on the cultural and political context for EHR deployment, in: J.E. Demetriades, R.M. Kolodner, G.A. Christopherson (Eds.), Person-Centered Health Records - Toward Health-e-People. Health Informatics Series, Springer Science + Business Media Inc., 2005.

[14] O. Tamburis, M. Mangia, M. Contenti, G. Mercurio, A. Rossi Mori, The LITIS conceptual framework: measuring eHealth readiness and adoption dynamics across the Healthcare Organizations, Health Technol. 2 (June (2)) (2012) 97-112 http://rd.springer.com/article/10.1007/s12553-012-0024-5

[15] eHealth ERA - Towards the Establishment of a European eHealth Research Area (2007). Deliverables. http://www.eHealth-era.org/index.htm

[16] A. Rossi Mori, M. Mazzeo, S. D'Auria, Deploying Connected Health among the Actors on Chronic Conditions, Eur. J. ePractice (8) (December 2009) http://www.epractice.eu/files/European\%20Journal \%20epractice\%20Volume\%208_1.pdf

[17] Centers for Medicare and Medicaid Services, Medicare and medicaid programs (CMS), Electronic Health Record Incentive Program (2010) http://cms.gov/EHrIncentivePrograms/

[18] A. Rossi Mori, R. Dandi, "The Influence of Technology on Long-Term Care Systems”. ENEPRI Policy Brief No. 10. 
European Network of Economic Policy Research Institutes, Bruxelles, February 2012. http://www.ceps.be/ category/book-series/enepri-policy-briefs

[19] A. Schmidt, F. Barbabella, F. Hoffmann, G. Lamura. "Analysis and Mapping of 52 ICT-based initiatives for family caregivers" CARICT Project, Deliverable 2.3, European Centre for Social Welfare Policy and Research, 2011.

[20] ANCIEN Consortium. "The technological solutions potentially involved in LTC activities". Deliverable D-IV.1, 2011.

[21] Recommendations agreed by the participants to the Special Topic Conference 2003 of the European Federation of Medical Informatics, Roma, 6th-7th October 2003. "The Content of the Electronic Health Record: Clinical Datasets for Continuity of Care and Pathology Networks," 2003. http://www.prorec.it/efmiStc/EFMI-STCrecommendations02c.doc

[22] A. Rossi Mori, F. Consorti, Integration of clinical information across patient records: a comparison of mechanisms used to enforce semantic coherence, IEEE Transactions on Information Technology in Biomedicine 2 (4) (1998) 243-253.

[23] A. Rossi Mori, F. Consorti, E. Galeazzi, Standards to support development of terminological systems for healthcare telematics, Meth. Inform Med. 37 (1998) 551-563.

[24] A. Rossi Mori, F. Consorti, E. Galeazzi, P. Merialdo, A second generation of terminological systems is coming, in: C. Pappas, et al. (Eds.), Medical Informatics Europe '97, IOS Press, Amsterdam, 1997, pp. 436-440.

[25] A. Rossi Mori, F. Consorti, Exploiting the terminological approach from CENTC251 to support interoperability of health record systems, Int. J. Med. Inf. 48 (1-3) (1997) 111-124.

[26] openEHR Foundation "EN13606 - a Standard for EHR System Communication". http://www.openehr.org/standards/cen.html

[27] [7HL] "Detailed Clinical Models". http://wiki.hl7.org/index.php?title=Detailed_Clinical_Models

[28] PCAST Report Workgroup, "Realizing the Full Potential of Health Information Technology to Improve Healthcare for Americans: The Path Forward", 2011. http://ahier.blogspot. com/2011/01/pcast-report-workgroup-1-14-2011.html

[29] Wikipedia "Health Level 7". http://en.wikipedia.org/wiki/Health_Level_7

[30] CEN TC251, "CONTSYS - A System of Concepts to Support Continuity of Care" EN 13940, European Standardization Committee (CEN), Brussels, 2006.

[31] M. Hägglund, R. Chen, S. Koch, Modeling shared care plans using CONTsys and openEHR to support shared home care of elderly, JAMIA 1 (18(1)) (2011) 66-69.

[32] M. Hägglund, M. Henkel, J. Zdravkovic, P. Johannesson, I. Rising, I. Krakau, S. Koch, A new approach for goal-oriented analysis of healthcare processes, Stud. Health Technol. Inform. 160 (2010) 1251-1255.

[33] M. Hägglund, I. Scandurra, S. Koch, Scenarios to capture work processes in shared homecare - from analysis to application, Int. J. Med. Inform. 79 (6) (2010) e126-e134.

[34] M. Hägglund, I. Scandurra, S. Koch, Studying points of intersection - an analysis of information needs in shared homecare of elderly, J. Inform. Technol. Healthcare 7 (1) (2009) 1-20.

[35] A. Rossi Mori, Cooperative development of the healthcare infostructure for Europe, in: Kevin Dean (Ed.), Connected Health. Thought Leaders. Essays from Health Innovators, Premium Publishing, London, 2003.

[36] A. Rossi Mori, et al., eHealth deployment roadmap and roll-out planning: Guiding design principles, in: Proceedings of the "eHealth Planning and Management Symposium 2008" - EuroRec Annual Conference 2008-11-03 Copenhagen in a joint meeting with EHTEL, 2008

http://www.ehtel.org/forum/conferences/ehealth-planning

[37] A. Rossi Mori, G. Mercurio, W. Palumbo, I. Paolini, L. Ruotolo, Focused profiles for chronic patients in integrated care and clinical governance, in: 9th International HL7 Interoperability Conference - IHIC 2008, Crete, Greece, 2008 http://www.hl7.org.gr/ihic2008/9o_congress/ihic_2008.html

[38] Wikipedia, "Clinical Document Architecture". http://en.wikipedia.org/wiki/Clinical_Document_Architecture

[39] Wikipedia, "Continuity of Care Document". http://en.wikipedia.org/wiki/Continuity_of_Care_Document

[40] UK Department of Health, "Improving Chronic Disease Management", 2004. http://www.dh.gov.uk/prod_consum dh/groups/dh_digitalassets/@dh/@en/documents/ digitalasset/dh_4075213.pdf

[41] EHTEL - Task Force Sustainable Telemedicine \& Chronic Disease Management. http://www.ehtel.org/activities/ tasks-sources/task-force-sustainable-telemedicine-andchronic-disease-management/

[42] E.H. Wagner, et al., Improving Chronic Illness Care: Translating Evidence Into Action, JAMA 288 (October 2002) 14.

[43] World Health Organization, Constitution of the World Health Organization". Basic Documents, forty-fifth ed., WHO, Geneva, October 2006 (Supplement).

[44] T. Østbye, et al., Is there time for management of patients with chronic diseases in primary care? Ann. Fam. Med. 3 (3) (2005).

[45] M. Rigby, Health informatics as a tool to improve quality in non-acute care - new opportunities and a matching need for a new evaluation paradigm, Int. J. Med. Inf. 56 (1999) 141-150.

[46] UK Department of Health. "The WSD Programme Headlines Findings", December 2011. http://www.dh.gov.uk/dr_ consum_dh/groups/dh_digitalassets/documents/digitalasset/ dh_131689.pdf

[47] Map of Medicine Ldt. "Map of medicine." http://www.mapofmedicine.com/

[48] Consejeria de Salud, Junta de Andalucia. "Procesos Asistenciales Integrados". http://www.juntadeandalucia.es/salud/servicios/procesos/

[49] Declaration by the Members of the European Science Foundation Exploratory Workshop on Social Care Informatics and Holistic Health Care, Keele University, UK, July. "Social Care Informatics meets Health Care Informatics - A Holistic Citizen-Centric Vision for Information and Communication Technologies to Support Personal Health", 2010. http://iig.umit.at/dokumente/n29.pdf

[50] M. Rigby, P. Hill, S. Koch, Keeling D, Social care informatics as an essential part of holistic health care: a call for action, IJMI 80 (8) (2011) 544-554.

[51] eHealth Initiative, "Centering on the Patient: How Electronic Health Records Enable Care Coordination”, 2011. http://www.ehealthinitiative.org/issues/care-coordination/ care-coordination-report.html

[52] M. Rigby, et al., Integrated record keeping as an essential aspect of a primary care led service, Br. Med. J. 317 (1998) 579-582.

[53] A. Rossi Mori, Integrating Care for Chronic Conditions through a lifelong EHR, in: Proceedings of the International Conference "Improving Care for Chronic Conditions - the Added Value of eHealth", Rome, 10-11 October 2005, Jointly Organised by EHTEL, the National Research Council of Italy Institute for Biomedical Technology in Co-operation with ESQH and NIZW, 2005 http://www.ehtel.org/ forum/conferences/event-2005-eHealth-added-value

[54] A. Rossi Mori, Position Statement: "Connecting systems or connecting people?" Panel: "Ptolemaic vs. Copernican - How healthcare policies and re-organisation of care provision will 
influence the eHealth roadmaps", in: International Conference "Continuity, Collaboration, Communication: Challenges for Healthcare and Opportunities for eHealth", Rome, 24-25 May 2007, Jointly Organised by EHTEL, the National Research Council of Italy - Institute for Biomedical Technology in Co-operation with CPME, EFN, HOPE, PGEU, UEMS, 2007 http://www.ehtel.org/forum/conferences/ roma-24-maggio-2007

[55] A. Rossi Mori, Move Forwards with Continuity, Collaboration and Communication, in: HIMSS-EMEA eMessenger, Issue of 2008-01-10, 2008 http://emea.himss.org/ eNewsletters/archive/2008/10_January.htm

[56] Rossi Mori, et al. Vision for a Europe-wide Semantically Interoperable eHealth Infrastructure. Deliverable RIDE D.3.2.1, 2006.

[57] Kaiser Permanente "HealthConnect ${ }^{\circledR}$ Electronic Health Record”. http://xnet.kp.org/newscenter/ aboutkp/healthconnect/index.html

[58] US Department of Veterans Affairs. "My Health-e-Vet". http://www.myhealth.va.gov/

[59] Maccabi Institute for Health Services Research. "The Information System As A Tool For Efficient Organizational Administration". http://www.maccabi-research.org/ 144.html

[60] The Official Google Blog. "An update on Google Health and Google PowerMeter", 2011. http://googleblog.blogspot. com/2011/06/update-on-google-health-and-google.html (accessed 06.24.11).
[61] HIMSS, "State HIT Dashboard". http://www.himss.org/StateDashboard/

[62] Wikipedia, "Health Information Exchange". http://en.wikipedia.org/wiki/Health_information_exchange

[63] D.K. Nace, J. Gartland, Providing accountability: accountable care concepts for providers, Relay Health (2011) http://healthsystemcio.com/white-papers/providingaccountability-aco-concepts-for-providers/

[64] ANCIEN Consortium, "A short introduction to ANCIEN Assessing Needs of Care In European Nations". http://www.ancien-longtermcare.eu/

[65] M. Maggini, R. Raschetti, A. Rossi Mori, et al., Requisiti informativi per un sistema di gestione integrata del diabete mellito di tipo 2 nell'adulto: documento di indirizzo, Pensiero Scientifico Editore, Roma, 2008 http://www. epicentro.iss.it/igea/documenti/documentiIGEA.asp

[66] M. Mazzeo, P. Agnello, A. Rossi Mori, "Role and Potential Influence of Technologies on the Most Relevant Challenges for Long-Term Care". ENEPRI Research Report No. 113. European Network of Economic Policy Research Institutes, Bruxelles, June 2012. http://www.ceps.be/category/ bookseries/enepri-research-reports

[67] A. Rossi Mori, F.L. Ricci, et al. "Report on Priority Topic Cluster One and recommendations: Patient Summary". Deliverable D 2.3 of the European Project "eHealth ERA Towards the Establishment of a European eHealth Research Area", Revision 1, February 2007. http://www.ehealthera. org/publications/publications.htm 